EPJ Web of Conferences 112,04004 (2016)

DOI: $10.1051 /$ epjconf/201611204004

C) Owned by the authors, published by EDP Sciences, 2016

\title{
Multiplicity distributions and fluctuations in proton-proton and heavy-ion collisions
}

\author{
Maitreyee Mukherjee ${ }^{a}$ \\ Variable Energy Cyclotron Centre, Kolkata, India
}

\begin{abstract}
The charged particle multiplicity is one of the simplest observable in collision of the hadrons, imposing constrains on the mechanisms of the particle production. Multiplicity distributions at lower energies have been studied earlier. In this work, it has been investigated how the multiplicity distributions and fluctuations have been explained in LHC energies. For this, a detailed study has been done from $\sqrt{s}_{s}=7.7 \mathrm{GeV} / \mathrm{A}$ to $\sqrt{s}_{\mathrm{s}}=2.76 \mathrm{TeV} / \mathrm{A}$ with event generators AMPT-Default and AMPT-String Melting. It will be also discussed how multiplicity distribution parameters are related to early stages of collision and how small-x helps to find information on initial states.
\end{abstract}

\section{Introduction}

Multiplicity, i.e, the number of charged particles produced per event is an important ingredient for the understanding of multi-particle production. Multiplicity of produced particles is an important quantity to characterize the evolving system. This is one of the very basic studies as many of the observables such as energy-density, transverse energy, momentum etc. are connected to the number of particles and its fluctuation event-by-event. Multiplicity is a global observable, and multiplicity distribution and fluctuation sheds light into mechanisms of particle production and provide constraints to the many models aiming at describing these collisions, it helps to reject or improve models $[1,2]$.

Because of the production of large number of particles in each heavy-ion collision at RHIC as well as LHC, it has become possible to make precision event-by-event measurements of several quantities and study their event-by-event fluctuations. QCD phase transition can manifest itself by characteristic behavior of several observables which may vary dramatically from one event to the other. Fluctuations are usually characterized by scaled variances. Dynamical fluctuations (other than the statistical fluctuation and fluctuation in the number of participants) may provide insights on the intrinsic mechanisms of the particle production.

Event-by-event multiplicity fluctuations are basically connected to QCD-phase transition. Variance of the multiplicity distributions are directly related to the isothermal compressibility $\left(\mathrm{k}_{\mathrm{T}}\right)$ of the system formed in heavy ion collision, i.e,

$$
\sigma^{2}=\frac{k_{B} T<N>^{2}}{V} k_{T}
$$

where, $\mathrm{k}_{\mathrm{B}}$ is Boltzmann's constant and $<\mathrm{N}>$ is the mean multiplicity. $\mathrm{k}_{\mathrm{T}}$ is expected to increase by at least an order of magnitude close to the QCD critical point [3]. Additionally, determination of critical

\footnotetext{
${ }^{a}$ Corresponding author: maitreyee.mukherjee@gmail.com
} 
exponent helps to determine the universality class in which QCD is grouped. Multilicity fluctuation expressed in terms of scaled variance, is basically the variance scaled over mean of the multiplicity distribution, which also is expected to increase by an order of magnitude near the QCD critical point.

\section{Earlier measurements}

Multiplicity distributions in $\mathrm{e}^{+} \mathrm{e}^{-}$collisions at $\sqrt{\mathrm{s}}=29 \mathrm{GeV}$ are described by Poisson distributions, indicates no correlation between the produced particles. $\mathrm{p}+\mathrm{p}$ non-single diffractive events behave little differently, follow dispersion $\mathrm{D} \infty \sqrt{ }<\mathrm{n}>$ upto max. ISR energy at $\sqrt{\mathrm{s}}=62 \mathrm{GeV}$ (where, $<\mathrm{n}>$ denotes mean multiplicity). In 1972, concept of KNO-scaling comes, which is an universal scaling followed at higher energies. Here, we get, $<n>\infty \ln \sqrt{s}[2]$. UA5 shows that KNO-scaling breaks at $\sqrt{s}_{\mathrm{s}}=200 \mathrm{GeV}$. This is shown in Fig 1.

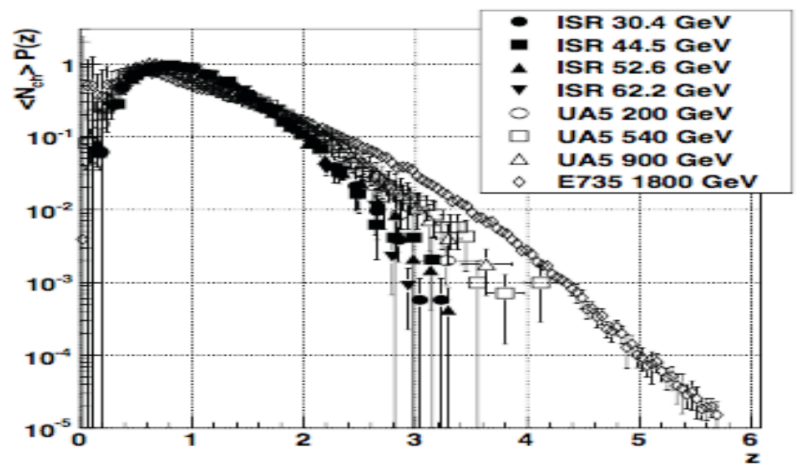

Figure 1. KNO-Scaling and its breaking

UA5 found that, upto $\sqrt{s}_{\mathrm{s}}=540 \mathrm{GeV}$, multiplicity distributions follow NBD, which has two parameters $<\mathrm{n}>$ and $\mathrm{k}$ (related to width), and $1 / \mathrm{k}$ is approximately proportional to $\ln \sqrt{\mathrm{s}}_{\mathrm{s}}$. Here, we can write the multiplicity distributions follow $[3,4]$,

$$
P(n)=\frac{\Gamma\left(n+k_{N B D}\right)}{\Gamma(n+1) \Gamma\left(k_{N B D}\right)} \frac{\left(\mu_{c h} / k_{N B D}\right)^{n}}{\left(1+\mu_{c h} / k_{N B D}\right)^{n+k_{N B D}}}
$$

where,

$$
\mu_{c h}=<N_{c h}>
$$

and,

$$
\sigma^{2}=\mu+\mu^{2} / k_{N B D}
$$

and, the scaled variance $\left(\omega_{\mathrm{ch}}\right)$ is given by,

$$
\omega_{c h}=1+\frac{\mu_{c h}}{k_{N B D}}
$$

Deviations from single NBD observed around $\sqrt{s}_{\mathrm{s}}=900 \mathrm{GeV}$. A shoulder-like structure seen. Thus concept of double NBD comes, where the distributions are described as the combination of two NBD's, one having soft component and another having the semi-hard component. Another description was given from the point of view of multi-parton interaction, which becomes very much important at higher energies. 


\section{Multiplicity fluctuations : Results from AMPT}

For this work, centrality has been selected using minimum-bias distribution of number of participants $\left(\mathrm{N}_{\text {part }}\right)$ as shown in Fig 2. $\mathrm{N}_{\text {part }}$ is directly proportional to some quantities that are experimentally observed, such as $\mathrm{N}_{\mathrm{ch}}$. So, centrality selection from $\mathrm{N}_{\text {part }}$ is quite justified. $\mathrm{N}_{\mathrm{ch}}$-minimum bias distribution is the convolution of $\mathrm{N}_{\mathrm{ch}}$-distributions in different centralities.

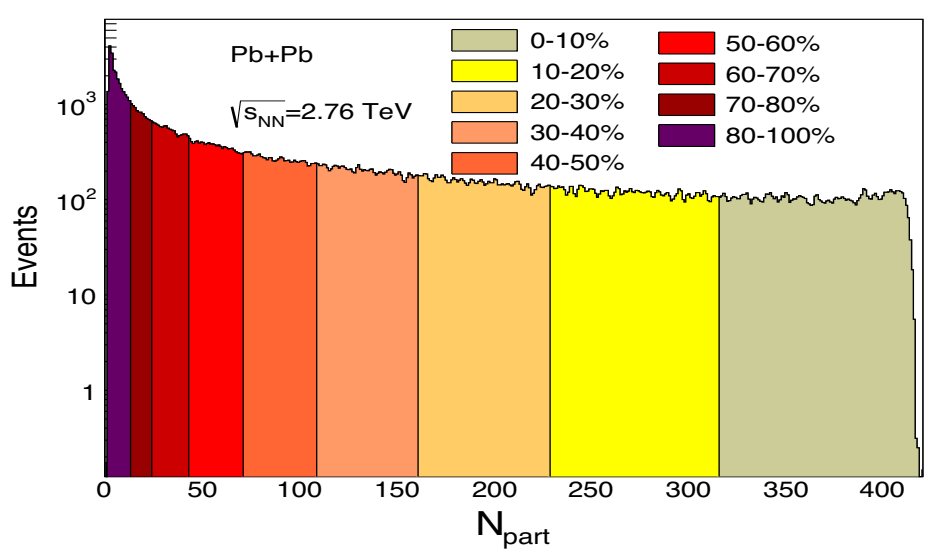

Figure 2. Centrality selection from $\mathrm{N}_{\text {part }}$-distribution using AMPT-model

Finer bin in centrality needs to be selected for fluctuation studies. This will avoid inherent fluctuations in $\mathrm{N}_{\text {part }}$ within a given centrality. In this analysis, we select $0-1 \%, 1-2 \%, 2-3 \%, 3-4 \%, \ldots \ldots$ centrality bins and also check with centrality binwidth corrections. Now, centrality bin-width effect arises due to the impact parameter(or volume) variations due to the finite centrality bin. To correct for this effect, the best way is to divide one centrality bin into smaller bins and weight the moments [5]. Fig 3 illustrates the correction due to the centrality binwidth-effect.

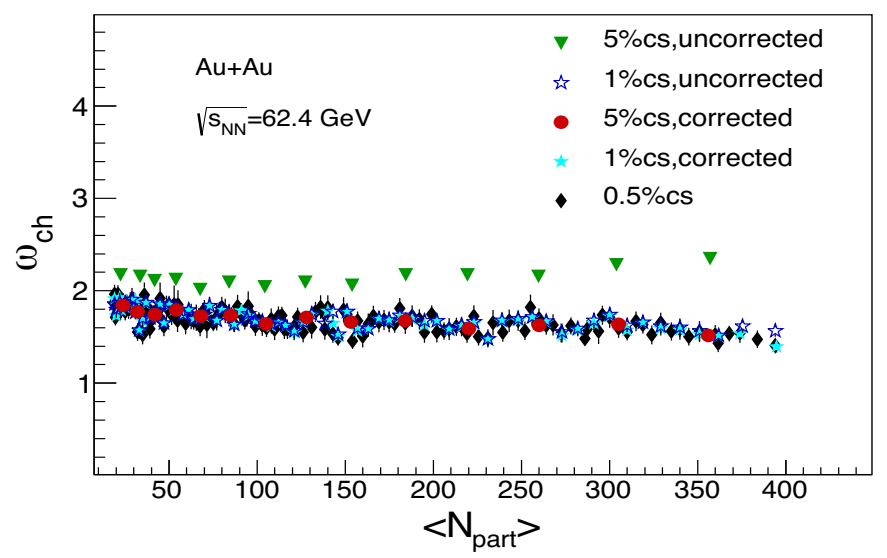

Figure 3. Centrality binwidth-effect

From Fig 3, it is evident that, the binwidth correction changes result for $5 \%$ cs by $\sim 25 \%$ and for $1 \%$ cs by $\sim 2-3 \%$ at the most central. So, doing analysis using narrower centrality bins(here, $0.5 \% \mathrm{cs}$ ) is quite 
The centrality dependence of the multiplicity fluctuation has been investigated in this work. The whole work has been done using AMPT-default and AMPT-String Melting model. The transverse momentum and pseudorapidity range is selected as $0.2<\mathrm{p}_{\mathrm{T}}<2 \mathrm{GeV} / \mathrm{c}$ and $|\eta|<0.5$, respectively. The result has been shown in Fig 4.

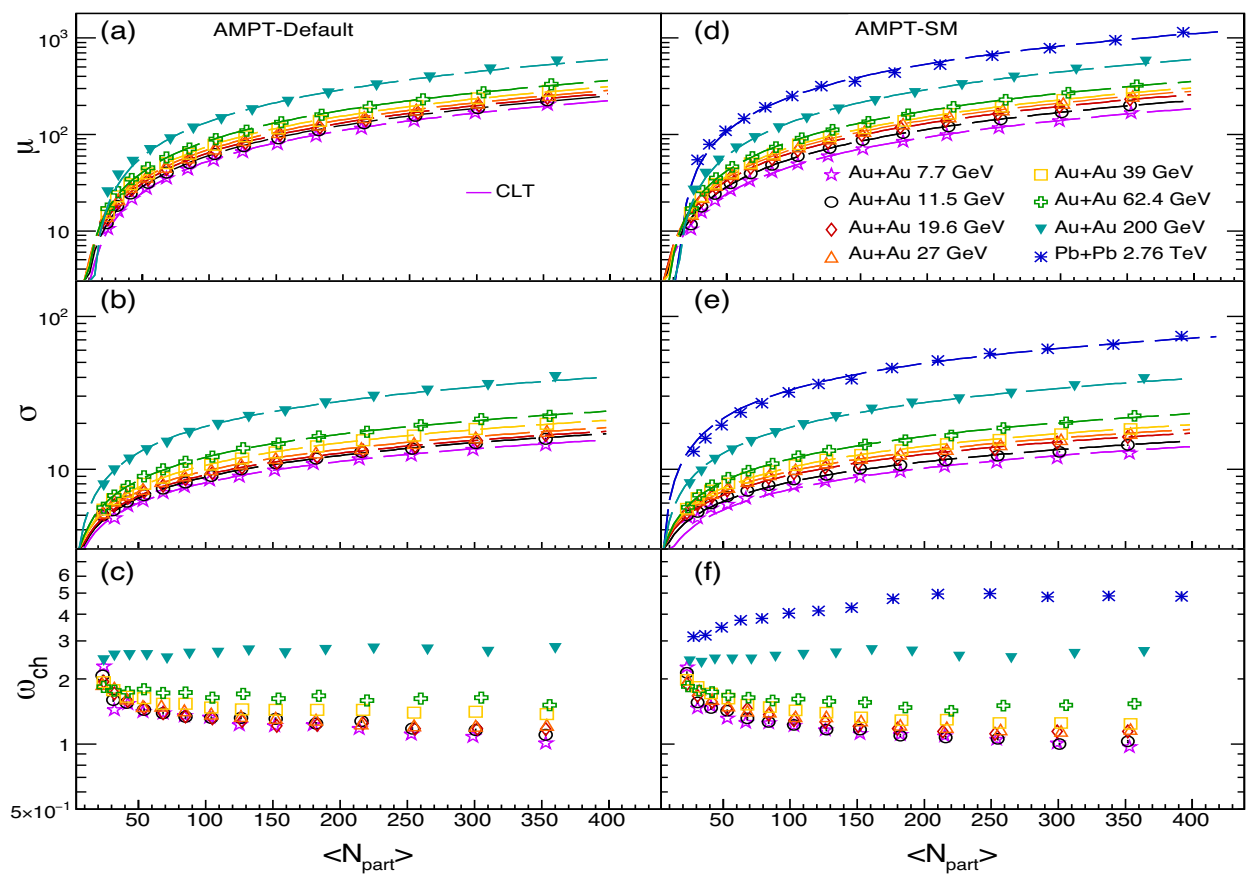

Figure 4. Centrality dependence of mean, variance and the scaled variance

We have observed that, Central Limit Theorem (CLT)-fits are better for $\mu$ and $\sigma$ for lower energies. For energies from $7.7 \mathrm{GeV}$ to $200 \mathrm{GeV}, \omega_{\text {ch }}$ shows increasing trend for central to peripheral collisions. For $2.76 \mathrm{TeV}$, the trend for scaled variance is somehow opposite. Result for AMPT-default has not been shown for $2.76 \mathrm{TeV}$, as we have observed that, results from String-Melting model are more reliable in such higher energies.

The beam-energy dependence of the scaled variance has also been investigated, which is shown here in Fig 5.

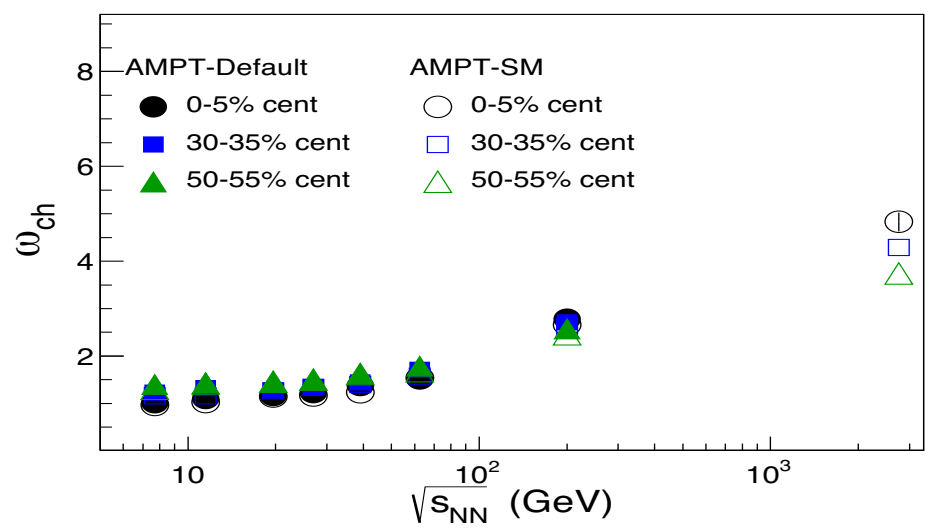

Figure 5. Beam-energy dependence of the scaled variance 
We observe that the fluctuations show an increasing trend from lower to higher energies. Also, the fluctuations at lower energies are close to each other for all centralities.

\section{Recent results from LHC}

The ALICE Collaboration has carried out a detailed study of multiplicity distributions of primary charged particles produced in proton-proton collisions, at $\sqrt{ } s=0.9,2.36,2.76,7$ and $8 \mathrm{TeV}[6]$ (Result for $8 \mathrm{TeV}$ is shown in Fig 6)

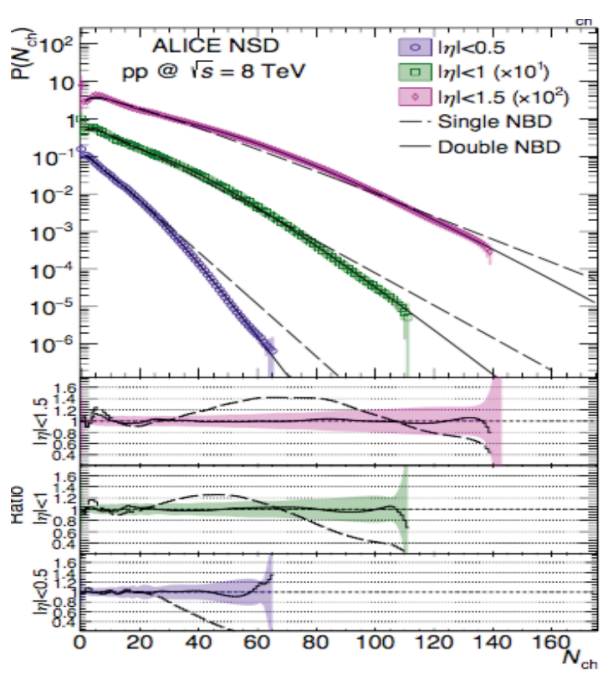

Figure 6. Multiplicity Distributions for NSD events

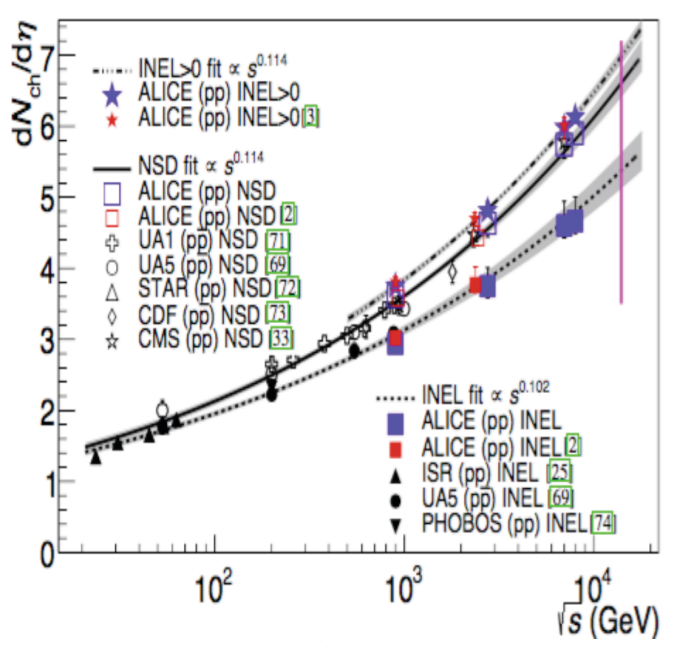

Figure 7. Charged-particle pseudorapidity density

It has been observed from Fig 6 that, at LHC energies, above $2 \mathrm{TeV}$, multiplicity distributions can no longer be represented by a single NBD, but a double NBD give a good representation of the data. Fig 7 shows that, the power law parameterization of $\mathrm{d} N_{\mathrm{ch}} / \mathrm{d} \eta$ at $\eta=0, s^{\delta}$, provides a good description of the data from ISR to LHC energies: $\delta=0.102 \pm 0.003,0.114 \pm 0.003$ and $0.114 \pm 0.001$, for the INEL, NSD and INEL $>0$ event classes, respectively [6]. The charged particle pseudorapidity density distribution has been measured in $\mathrm{Pb}-\mathrm{Pb}$ collisions at $\sqrt{ } s=2.76 \mathrm{TeV}$ also in ALICE [7]. The measurement was performed in a wide pseudorapidity interval of $-5.0<\eta<5.5$ allowing the total number of charged particles produced at the LHC. Results are shown in Fig 8 [7].

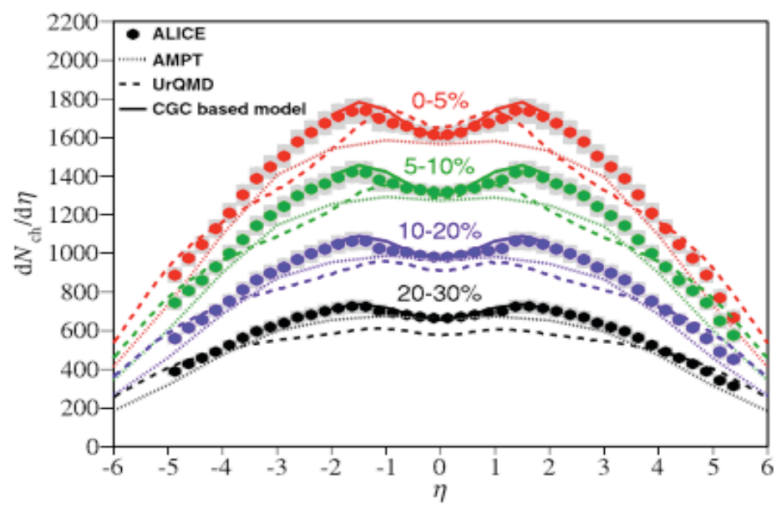

Figure 8. Comparison to models with data from ALICE 
We observe that the theoretical predictions do not describe the data well, except the CGC based model within its limited pseudorapidity range.

\section{Multiplicity distributions : connection to early stages of collision}

At small enough Bjorken-x, we get a saturation region, where the non-linear effects due to high gluon density becomes very important [8]. Small Bjorken-x (expressed as $x=p_{T} /(\sqrt{ } s) \cdot e^{-\eta}$ ) values can be accessed by going to higher center-of-mass energy and higher rapidity, which is easier to achieve in LHC energies. So, this is an opportunity to study initial state effects. Yang Mills dynamics gives framework to study correlated multi-particle production. From Glasma flux-tube picture, NBD-k scaled over mean multiplicity can be expressed as $[9,10]$,

$$
\frac{k}{\bar{n}}=\frac{2 N_{c} \pi}{c}\left(\kappa \alpha_{s}\right)
$$

where, $\mathrm{N}_{\mathrm{c}}$ are the number of gluon-colors, $\mathrm{c}$ is a constant and $x$ is a non-perturbative constant. So, we

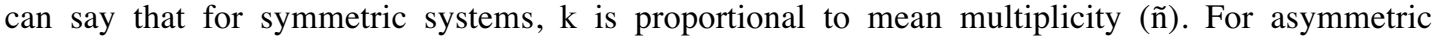
systems (say, p-Pb), we have [11],

$$
\bar{n} \sim k \ln ^{2} \frac{Q_{s, P b}^{2}}{Q_{s, p}^{2}}
$$

where, $\mathrm{Q}_{\mathrm{s}}$ denotes the saturation-momentum. We see, this is not a proportional-dependence. Thus, we can differentiate between symmetric and asymmetric system. Thus, the multiplicity distribution parameters are related to early stages of collision.

\section{References}

1. M. Gazdzicki, On the history of multiparticle production in high energy collisions, Acta Phys.Polon.B 43 (2012) 791; arXiv:1201.0485 [hep-ph] (2012).

2. J. Fiete Grosse-Oetringhaus, K.Reygers, J.Phys. G 37083001 (2010).

3. PHENIX Collaboration, A. Adare et al., Phys. Rev. C 78, 044902 (2008).

4. WA98 Collaboration, M.M. Aggarwal et al., Phys. Rev. C 65, 054912 (2002).

5. N.R. Sahoo, S. De, T.K. Nayak, Phys.Rev. C 87, 044906 (2013).

6. ALICE Collaboration, J. Adam et al., arxiv : 1509.07541 [nucl-ex] (2015).

7. ALICE Collaboration, E. Abbas et al., Physics Letters B 726 (2013) 610-622.

8. E. Iancu, arxiv : 1205.0579 [hep-ph] (2012).

9. B. Schenke, P. Tribedy, R. Venugopalan, Phys. Rev. C 86 (2012).

10. T. Lappi, arxiv : 1104.3725 [hep-ph] (2011).

11. A.H.Rezaeian, arxiv : 1308.4736 [hep-ph] (2013). 